\title{
Resistive stability of a plasma with runaway electrons
}

\author{
P. Helander ${ }^{1}$, D. Grasso ${ }^{2}$, R.J. Hastie ${ }^{3}$, A. Perona ${ }^{2}$ \\ ${ }^{1}$ Max-Planck-Institut für Plasmaphysik, 17491 Greifswald, Germany \\ ${ }^{2}$ Politecnico di Torino, Dipartimento di Energetica, 10129 Torino, Italy \\ ${ }^{3}$ Euratom/UKAEA Fusion Association, Culham Science Centre \\ Abingdon OX14 3DB, U.K.
}

In tokamak disruptions the Ohmic current is often replaced by a current of runaway electrons, which is likely to be more peaked in the centre of the discharge than the pre-disruption current. This raises the question of the resistive stability of the post-disruption plasma, where the equilibrium current is entirely carried by the runaway electrons while the cold $(\sim 10 \mathrm{eV})$ background plasma is relatively resistive. It is found that the linear properties of the classical tearing mode are essentially determined by the cold bulk plasma, and the growth rate is approximately the same as in a plasma without runaways but with the same current profile. The nonlinear saturation amplitude is different however. In a symmetric plasma slab, the saturated island size is larger when the current is carried by runaway electrons than in the Ohmic case, and undergoes a nonlinear bifurcation when the stability index $\Delta^{\prime}$ exceeds a critical value.

PACS numbers: 52.35.Py, 52.55.Tn, 52.55.Fa 


\section{Introduction}

When a tokamak plasma undergoes a disruption, it is quickly cooled to a temperature of around $10 \mathrm{eV}$ and a significant fraction of the pre-disruption current is converted to a current of highly relativistic "runaway" electrons. This fraction is typically around $1 / 2$ in the Joint European Torus (JET), for reasons that have only recently been understood theoretically [1]. The runaway electrons are produced by the toroidal electric field which is induced as the plasma cools down during the thermal quench of the disruption. Because the resistive diffusion time after the thermal quench is comparable to the generation time for runaways (the inverse runaway avalanche growth rate [2]), some of this electric field diffuses to the wall and leaves the plasma before producing any runaways. How much of the pre-disruption current is converted to runaways is thus governed by a competition between resistive diffusion of toroidal electric field and runaway avalanche growth. More runaways are predicted to be produced in ITER than in JET, since the avalanche is stronger and the skin time is longer.

Another prediction of the theory in Ref. [1] is that the post-disruption runaway current profile could be more strongly peaked in the centre of the discharge than the predisruption current. This occurs because runaway electrons are most quickly produced in the hottest part of the plasma, i.e., in the centre, and once this has happened the radial profile of the toroidal electric field becomes hollow, causing inward diffusion of this field and resulting in more runaway production in the centre of the discharge and less further out. It appears that this phenomenon may have been observed experimentally on JET [3], although it was not recognised at the time.

Plasmas with steep current profiles are more prone to tearing-mode instability, which provides the motivation for the present paper - to investigate the resistive stability of a post-disruption plasma with runaway electrons. After a few skin times, there is very little toroidal electric field left in the plasma and practically all the current is carried by essentially collisionless runaway electrons. In contrast, the background plasma is highly resistive, and it is not immediatly clear what component determines the resistive stability properties, the collisionless runaways or the resistive bulk. We focus on the simplest case of an ordinary cylindrical tearing mode with poloidal mode number $m>1$ and completely collisionless runaway electrons, with no cross-field magnetic drift, traveling at the speed of light along the magnetic field. At least in this 
simple case, it turns out that it is the cold bulk plasma that controls the linear properties of the resistive layer. The linear instability threshold, mode structure and growth rate are approximately the same as if the current had been carried by thermal electrons. If $\Delta^{\prime}>0$ [defined in the usual way, see Eq. (31)] the mode is thus expected to grow rapidly in a $10 \mathrm{eV}$ plasma since the resistivity is very high. When the magnetic island associated with the instability has grown to a size much larger than the linear tearing layer width, it enters a stage of slower (algebraic rather than exponential) growth [8] before finally saturating at some finite amplitude. We find that the saturated width of the island is different from that in a purely thermal plasma. This is because the final size of the island is sensitive to the form of Ohm's law that governs the steady-state plasma current, and this is very different in a plasma with runaway electrons. Unlike the currents that arise during the growth of the instability, the final current is noninductive in nature and is exclusively carried by runaway electrons. Our calculation follows the general method laid down in Ref. [9], modified appropriately to allow for the fact that it is runaway electrons that carry the current determining the final shape and size of the island. These analytical predictions are borne out by the numerical calculations presented in Sec. V, where it is also found that there is a nonlinear bifurcation in the saturated island size at a critical value of $\Delta^{\prime}$. In principle this bifurcation could be calculated by the same analytical method by going to higher order in the $\Delta^{\prime}$-expansion. However, this calculation is sufficiently tedious that we have not carried it out but instead present a more approximate estimate of the critical island width at which the bifurcation occurs.

Before proceeding with the formal analysis, it is useful to make some simple estimates. The parallel resistivity of a $10 \mathrm{eV}$ hydrogen plasma with the number density $n_{e}=5 \cdot 10^{19} \mathrm{~m}^{-3}$ is

$$
\eta_{\|}=1.96 n_{e} e^{2} \tau_{e i} / m_{e} \simeq 18 \mu \Omega \mathrm{m},
$$

which is $10^{3}$ times that of copper and $50 \%$ larger than that of graphite. If the magnetic field is $B_{0}=3 \mathrm{~T}$, the Alfvén velocity is substantially smaller than the speed of light,

$$
v_{A}=B / \sqrt{\mu_{0} m_{i} n_{e}} \simeq 0.02 c,
$$

so the runaway electrons travel effectively infinitely fast along the magnetic field. The 
normalized resistive tearing mode layer width is

$$
w=(\gamma \eta)^{1 / 4} / k_{\|}^{\prime 1 / 2}
$$

where $\eta=\eta_{\|} R_{0} / \mu_{0} v_{A} a^{2}$ is the normalized resistivity, $\gamma$ the growth rate normalized to the Alfvén time $\tau_{A}=R_{0} / v_{A}$, with $R_{0}$ the radius of the magnetic axis, and $k_{\|}^{\prime}=$ $n s / r$, with $n$ the toroidal mode number, $s$ the magnetic shear, and $r$ the minor radius normalized to $a$, which denotes the radius of the plasma column. For a conventional $m>1$ tearing mode the growth rate is

$$
\frac{\gamma}{\tau_{A}}=\frac{\eta^{3 / 5} k_{\|}^{\prime 2 / 5}}{\tau_{A}}\left[\frac{\Gamma(1 / 4) a \Delta^{\prime}}{2 \pi \Gamma(3 / 4)}\right]^{4 / 5} \simeq 2 \cdot 10^{3} \mathrm{~s}^{-1},
$$

and the layer width becomes

$$
w a \simeq 1 \mathrm{~cm},
$$

where we have used JET-like parameters: $R_{0}=3 \mathrm{~m}, a=1 \mathrm{~m}, s=0.5, r=0.2$, and assumed $n=a \Delta^{\prime}=1$. The layer is thus much wider than the ion gyroradius and can be expected to be well described by resistive MHD.

\section{Basic equations}

The plasma is taken to consist of two distinct components: a cold bulk governed by reduced resistive MHD equations, and a population of highly relativistic runaway electrons moving at the speed of light along the magnetic field. The usual reduced MHD equations are at low beta [4]

$$
\begin{gathered}
\frac{\partial \psi}{\partial t}+\nabla_{\|} \phi=\eta \nabla_{\perp}^{2} \psi, \\
\frac{\partial \nabla_{\perp}^{2} \phi}{\partial t}+\left[\phi, \nabla_{\perp}^{2} \phi\right]+\nabla_{\|} \nabla_{\perp}^{2} \psi=0,
\end{gathered}
$$

where $\psi$ and $\phi$ are the normalized magnetic and electric potentials

$$
\begin{gathered}
\psi=\frac{A_{z}}{\epsilon B_{0} a}, \\
\phi=\frac{\Phi}{\epsilon B_{0} a v_{A}},
\end{gathered}
$$

$\epsilon=a / R_{0}$ and time has been normalized to the Alfvén time $\tau_{A}=R / v_{A}$. The Poisson brackets in Eqs. (3) and (4) are defined in the usual way,

$$
[A, B]=\frac{\partial A}{\partial x} \frac{\partial B}{\partial y}-\frac{\partial B}{\partial x} \frac{\partial A}{\partial y}
$$


and

$$
\begin{aligned}
& \nabla_{\perp}^{2} A=\frac{\partial^{2} A}{\partial x^{2}}+\frac{\partial^{2} A}{\partial y^{2}}, \\
& \nabla_{\|} A=\frac{\partial A}{\partial z}-[\psi, A],
\end{aligned}
$$

where $x=\left(R-R_{0}\right) / a, y=Z / a, z=-\zeta$, and $(R, \zeta, Z)$ are cylindrical coordinates defined relative to the symmetry axis of the torus.

In a plasma with runaway electrons, Ohm's law (3) must be modified to account for the runaway current. The latter needs to be subtracted from the right-hand side, so that the resistive term only contains the current carried by thermal electrons. Thus we replace Eq. (3) by

$$
\frac{\partial \psi}{\partial t}+\nabla_{\|} \phi=\eta\left(\nabla_{\perp}^{2} \psi+j\right)
$$

where $j$ is the normalized runaway current density. In the zero-gyroradius limit, the runaway electron distribution function $f$ is governed by the drift kinetic equation

$$
\frac{1}{\tau_{A}} \frac{\partial f}{\partial t}+\left(\frac{v_{\|} \mathbf{B}}{B}+\frac{\mathbf{E} \times \mathbf{B}}{B^{2}}\right) \cdot \nabla f=0
$$

The runaways move practically at the speed of light along the magnetic field, so it is straightforward to integrate over velocity space and obtain an equation for the normalized runaway current density $j$,

$$
\frac{\partial j}{\partial t}+[\phi, j]-\frac{c}{v_{A}} \nabla_{\|} j=0
$$

which closes the system of equations. Here we have taken the current to be positive, so that the runaway electrons move in the negative direction, $v_{\|}=-c$.

We now proceed to linearize these equations around an equilibrium described by $\psi=\psi_{0}(r), \phi_{0}=0$ and $j_{0}(r)=-\nabla_{\perp}^{2} \psi_{0}$, where $r^{2}=x^{2}+y^{2}$. Note that all the equilibrium current is carried by the runaway electrons. The normalized poloidal equilibrium magnetic field is equal to $-\psi_{0}^{\prime}(r)$ and the safety factor becomes $q(r)=-r / \psi_{0}^{\prime}(r)$. A Fourier transform of the perturbations is taken in the poloidal angle, $\theta$, the torodial angle, $z$, and in time, $t$, so that

$$
\psi_{1}(r, \theta, t)=\hat{\psi}(r) e^{\gamma t+i(n z-m \theta)},
$$

and we identify the parallel wave vector $k_{\|}(r)=n-m / q(r)$. Dropping carets over $\psi$, $\phi$ and $j$, and writing

$$
\hat{\nabla}_{\perp}^{2} A(r)=\frac{1}{r} \frac{d}{d r}\left(r \frac{d A}{d r}\right)-\frac{m^{2} A}{r^{2}}
$$


we then obtain the equations

$$
\begin{gathered}
\gamma \psi+i k_{\|} \phi=\eta\left(\hat{\nabla}_{\perp}^{2} \psi+j\right), \\
\gamma \hat{\nabla}_{\perp}^{2} \phi+i k_{\|} \hat{\nabla}_{\perp}^{2} \psi=-\frac{i m j_{0}^{\prime}}{r} \psi, \\
\left(k_{\|}+i \gamma v_{A} / c\right) j=\frac{m j_{0}^{\prime}}{r}\left(\psi+v_{A} \phi / c\right),
\end{gathered}
$$

which define our linear problem. Note that after Fourier transformation the equation (17) for the runaway current is no longer a differential equation.

\section{Linear stability}

The analysis of the linear stability properties is carried out in the customary way by assuming that the dimensionless resistivity is small, $\eta \ll 1$, and therefore only matters in a small region around the resonance $k_{\|}\left(r_{s}\right)=0$ which can thus be considered separately from the "outer" region away from the resonance [5]. Resistivity can be neglected in the outer region, where we obtain from Eq. (16)

$$
k_{\|} \hat{\nabla}_{\perp}^{2} \psi=-\frac{m j_{0}^{\prime}}{r} \psi
$$

if we anticipate that the growth is slow on the Alfvén time scale. Hence and from Eqs. (15) and (17) with $\eta=0$ we obtain

$$
j=-\hat{\nabla}_{\perp}^{2} \psi=\frac{j_{0}^{\prime} / r}{\frac{n}{m}-\frac{1}{q}} \psi
$$

and we conclude that the runaway electrons do not affect the behaviour of the outer region. Ideal MHD is oblivious of what plasma component carries the current as long as the current-carrying species is tied to the magnetic field. Note that we have neglected the magnetic drift of the runaway electrons.

The resistive layer, where $x=r-r_{s} \sim w \ll 1$, is more interesting. Here we make the usual approximations $k_{\|}=k_{\|}^{\prime} x, \hat{\nabla}_{\perp}^{2}=d^{2} / d x^{2}$ and obtain the equations

$$
\begin{gathered}
\gamma \psi+i k_{\|}^{\prime} x \phi=\eta\left(\psi^{\prime \prime}+j\right), \\
\gamma \phi^{\prime \prime}+i k_{\|}^{\prime} x \psi^{\prime \prime}=-i k_{\|}^{\prime} J^{\prime} \psi, \\
\left(k_{\|}^{\prime} x+i \gamma v_{A} / c\right) j=k_{\|}^{\prime} J^{\prime}\left(\psi+v_{A} \phi / c\right),
\end{gathered}
$$


with $J^{\prime}=m j_{0}^{\prime} / k_{\|}^{\prime} r=q^{2} j_{0}^{\prime} / q^{\prime} r$. It is immediately clear that if the equilibrium current gradient vanishes at the resonance, $j_{0}^{\prime}\left(r_{s}\right)=0$, then the runaway electrons have no effect on the problem since the runaway current perturbation $j$ then vanishes in the layer. On the other hand, if $j_{0}^{\prime}\left(r_{s}\right) \neq 0$, then $j$ becomes very large near $x=0$ since

$$
j=\frac{J^{\prime}\left(\psi+v_{A} \phi / c\right)}{x+i \gamma v_{A} / k_{\|}^{\prime} c}
$$

or approximately

$$
j \simeq \frac{J^{\prime} \psi}{x}
$$

where we have anticipated that $\gamma v_{A} / c \ll w$ and $v_{A} \phi / c \ll \psi$. In this approximation, which we shall adopt henceforth, the runaway current density is infinite at $x=0$. Equation (20) indicates that the magnetic potential has a singularity of the form $\psi \sim$ $\psi(0)\left(1+J^{\prime} x \ln |x|\right)$ at the origin.

It is useful to rescale the radial coordinate to the layer width (63) by writing $x=w z$ and to write Eqs.(20)-(22) as

$$
\begin{gathered}
\frac{d^{2} \psi}{d z^{2}}=\frac{\delta}{z}\left(i \frac{d^{2} \varphi}{d z^{2}}+\zeta \psi\right), \\
\frac{d^{2} \varphi}{d z^{2}}-z^{2} \varphi=-i z \psi
\end{gathered}
$$

where $\varphi=\phi \delta^{-1 / 2}, \zeta=-J^{\prime} \eta^{3 / 4} k_{\|}^{\prime 1 / 2} / \gamma^{5 / 4}$ is expected to be of order unity, and

$$
\delta=\frac{\gamma^{3 / 2}}{\eta^{1 / 2} k_{\|}^{\prime}} \ll 1
$$

is expected to be small (of order $w$ ) for $m>1$ tearing modes since $\gamma \sim \eta^{3 / 5}$, see Eq. (2). These equations are now solved by expanding $\psi=\psi^{(0)}+\psi^{(1)}+\cdots$ and $\varphi=\varphi^{(0)}+\varphi^{(1)}+\cdots$ in the small parameter $\delta$. The presence of runaway electrons gives rise to the term involving $\zeta$ in Eq. (23), which is small in most of the resistive layer but of order unity when $z \sim \delta$, i.e., in a sublayer of width $w^{2}$ around the resonant surface. The presence of this term also changes the equations in a qualitative way. Without runaways, there are two modes of definite parity: the tearing mode with even $\psi$ and odd $\varphi$, and the twisting mode with odd $\psi$ and even $\varphi$. The presence of runaway electrons mixes these parities (weakly, as we shall see). As in the conventional treatment of this problem, Eq. (23) yields in lowest order for $z \sim 1$

$$
\frac{d^{2} \psi^{(0)}}{d z^{2}}=0 \quad \Rightarrow \quad \psi^{(0)}=A+B z
$$


which is the basis for the "constant- $\psi$ approximation" of Ref. [5]. In the sublayer, $z \sim \delta$, this solution needs to be modified. Here

$$
\frac{d^{2} \psi^{(0)}}{d z^{2}}-\frac{\delta \zeta \psi^{(0)}}{z}=\left.\frac{\delta i}{z} \frac{d^{2} \varphi}{d z^{2}}\right|_{z=0},
$$

so that

$$
\psi^{(0)}=2 \sqrt{\delta \zeta z}\left[c_{1} K_{1}(2 \sqrt{\delta \zeta z})+c_{2} I_{1}(2 \sqrt{\delta \zeta z})\right]-\left.\frac{i}{\zeta} \frac{d^{2} \varphi}{d z^{2}}\right|_{z=0}
$$

for $z>0$ and

$$
\psi^{(0)}=2 \sqrt{-\delta \zeta z}\left[c_{3} Y_{1}(2 \sqrt{-\delta \zeta z})+c_{4} J_{1}(2 \sqrt{-\delta \zeta z})\right]-\left.\frac{i}{\zeta} \frac{d^{2} \varphi}{d z^{2}}\right|_{z=0}
$$

for $z<0$. Using small-argument expansions of the Bessel function and choosing the coefficients to match across $z=0$ gives

$$
\psi^{(0)} \simeq c_{0} z+c_{1}(1+\delta \zeta z \ln |\delta \zeta z|),
$$

This solution matches Eq. (25) in the overlap region $\delta \ll z \ll 1$, if we choose $c_{0}=B$ and $c_{1}=A$. Note that the additional, logarithmic term in (29) is small everywhere in the resistive layer but has a large second derivate in the sublayer, $z \sim w$. As noted in Eq. (22), the current density is infinite at $x=0$ in the approximation we have adopted. This singularity is resolved by the finite value of $\gamma v_{A} / c$.

This "inner" solution of the resistive layer should match smoothly to the outer MHD region, where Eq. (19) implies

$$
\psi \simeq a_{1}\left(1-J^{\prime} x \ln |x|\right)+a_{ \pm} x
$$

in the matching region, $w \ll x \ll 1$. The coefficient of the last term is, in general, different for $x>0$ and $x<0$, the difference being

$$
\Delta^{\prime}=\frac{a_{+}-a_{-}}{a_{1}}=\lim _{z \rightarrow+\infty} \frac{\psi^{\prime}(z)-\psi^{\prime}(-z)}{w \psi(0)} .
$$

It is reassuring that when the constant terms of Eqs. (29) and (30) are matched, $a_{1}=c_{1}$, the logarithmic terms match automatically $[6,7]$. The requirement that the linear terms should match implies that $c_{1}$ must be small, $c_{1}=O(\delta)$. Although the presence of runaways mixes parities, $\psi$ is thus nevertheless approximately even. The jump $\Delta^{\prime}$ in the slope of the outer solution has no counterpart in the lowest-order inner solution $\psi^{(0)}$ and is instead obtained from the next order layer equation (23), which is

$$
\frac{d^{2} \psi^{(1)}}{d z^{2}}=\frac{\delta i}{z} \frac{d^{2} \varphi^{(0)}}{d z^{2}}
$$


in the region $z \sim 1$. Integrating this equation across the layer gives

$$
\Delta^{\prime}=\frac{\delta i}{w A} \int_{-\infty}^{\infty} \frac{d^{2} \varphi^{(0)}}{d z^{2}} \frac{d z}{z}
$$

Here $\varphi^{(0)}$ is obtained from $(24)$

$$
\frac{d^{2} \varphi^{(0)}}{d z^{2}}-z^{2} \varphi^{(0)}=-i z \psi^{(0)} \simeq-i A z .
$$

Only the part of $\psi$ that is even in $z$ contributes to $\Delta^{\prime}$, so in order to calculate this quantity we need to include the odd part of $\varphi^{(0)}$ on the right-hand side of Eq. (42), and hence only the even part of $\psi^{(0)}$ in Eq. (34). According to Eq. (29) this even part is a constant to the requisite order, and it follows that Eq. (34) reduces to that solved in conventional tearing mode theory, with a solution that can be expressed in terms of parabolic cylinder functions, see the Appendix and Ref. [10].

Thus we conclude that both the mode structure of the tearing layer, as described by $\psi(z)$ and $\varphi(z)$, and boundary condition, encapsulated in $\Delta^{\prime}$, are only weakly affected by the presence of runaway electrons in the plasma. The linear properties of the tearing mode are thus similar to those in a plasma without runaways to leading order in the smallness of $\eta$. It is well know in the conventional theory of tearing modes, however, that the growth rate (2) is only attained at very small values of $\eta$, making it rather difficult to reproduce this growth rate by direct numerical simulation. Since such simulations are carried out at finite resistivity, higher-order corrections tend to be important [7]. One would think that these corrections are also relatively important in post-disruption plasmas, because of their low temperature and resistivity. As shown in the appendix, however, the leading-order correction due to finite resistivity is actually absent in a plasma where the current is carried entirely by runaway electrons. This result will be confirmed numerically in Sec. V.

\section{Nonlinear saturation}

As is well known in tearing-mode theory, the exponential growth of a linearly unstable tearing mode is followed by a stage of slower, algebraic growth [8], eventually leading to saturation. We now consider the problem of calculating the nonlinear saturation amplitude in a runaway plasma. Following early work on this subject $[11,12]$, there has been a flurry of recent activity in this field $[9,13,14,15,16]$. All these works considered 
the question of what size the island grows to in the case when the equilibrium current is Ohmic. Here we consider the corresponding problem when the equilbrium current is instead carried by runaway electrons. For simplicity, we restrict our attention to the simplest geometrical case of a symmetric slab equilibrium, which we describe in Cartesian coordinates $(x, y)$, assuming that the equilbrium current density $j_{0}(x)$ has a maximum at the location of the resonant surface, $x=0$.

We begin by noting that although Ohmic currents exist in the plasma during the linear and nonlinear growth of the mode, in the final state all the current is again carried by runaway electrons. As long as the mode grows, there is an inductive electric field [the first term in Eq. (10)] driving a current in the resistive bulk plasma. But when the mode has saturated and the plasma has settled down in a new equilibrium (with a magnetic island), no inductive field or Ohmic current can exist and all that remains is the runaway current. In order to calculate the size of the magnetic island, we need to relate this final current profile,

$$
j_{\dagger}(x, y)=-\nabla_{\perp}^{2} \psi_{\dagger}(x, y)
$$

to the "initial" runaway current profile before the onset of tearing instability,

$$
j_{0}(x)=-\nabla_{\perp}^{2} \psi_{0}(x)
$$

Both these current densities are flux functions of the corresponding magnetic fields, i.e., they can be written as $j_{0}(x)=F_{0}\left[\psi_{0}(x)\right]$ and $j_{\dagger}(x, y)=F_{\dagger}\left[\psi_{\dagger}(x, y)\right]$, since Eq. (12) implies $\nabla_{\|} j=0$ to leading order in $v_{A} / c \ll 1$. We find the relation between $\psi_{0}(x)$ and $\psi_{\dagger}(x, y)$ by using two conservation laws. The first one is that of incompressibility. Since we are operating in slab geometry with a strong guide field, the $E \times B$ velocity is incompressible and the volume occupied by each fluid element is the same before and after the island has formed. The second conservation law is the condition that the runaway current remains "frozen into" the magnetic field during the evolution of the island, implying that $F_{0}=F_{\dagger}$. The freezing-in condition follows from the circumstance that the runaway electrons are convected by the $E \times B$ velocity, and is seen formally by noting that Eq. (12) implies

$$
\frac{\partial j}{\partial t}+\langle[\phi, j]\rangle=0
$$


where we have performed an average over the perturbed flux surfaces, denoted by angular brackets. This is defined as the volume average between two neighbouring flux surfaces,

$$
\langle\cdots\rangle=\oint\left|\frac{\partial x}{\partial \psi}\right|_{y}(\cdots) d y / \oint\left|\frac{\partial x}{\partial \psi}\right|_{y} d y
$$

where the integral is taken over one period and thus annihilates the parallel derivative. Hence, in Eq.(37)

$$
\langle[\phi, j]\rangle=\frac{\partial j}{\partial \psi}\langle[\phi, \psi]\rangle=\frac{\partial j}{\partial \psi}\left\langle\nabla_{\|} \phi\right\rangle=0
$$

so that

$$
\left\langle\left(\frac{\partial j}{\partial t}\right)_{x, y}\right\rangle=\left(\frac{\partial j}{\partial t}\right)_{\psi}+u(\psi, t)\left(\frac{\partial j}{\partial \psi}\right)_{t}=0
$$

where $u(\psi, t)=\langle\partial \psi / \partial t\rangle$. The physical interpretation of this relation is that the runaway current is "frozen" into the magnetic field.

It was found in Refs. $[13,14,15]$ that, as long as the island is small enough, it remains of approximately the same shape as the linear island, so we may write

$$
\begin{gathered}
\psi_{0}(x) \simeq \frac{\psi_{0}^{\prime \prime} x^{2}}{2} \\
\psi_{\dagger}(x, y) \simeq \psi_{0}(x)-\tilde{\psi}(\cos k y-1),
\end{gathered}
$$

reducing our task to that of calculating the nonlinear saturation amplitude $\tilde{\psi}$. It is convenient to normalize the $x$-coordinate to the island width $w$,

$$
X=\frac{x}{4} \sqrt{\frac{\psi_{0}^{\prime \prime}}{\tilde{\psi}}}=\frac{x}{w},
$$

and to introduce the flux-surface labels

$$
\begin{gathered}
\Omega(x, y)=\frac{\psi_{\dagger}(x, y)}{\tilde{\psi}}-1=8 X^{2}-\cos \theta, \\
m=\frac{\Omega+1}{2},
\end{gathered}
$$

so that $-1<\Omega<1$ and $0<m<1$ inside the island, while $1<\Omega<\infty$ and $1<m<\infty$ outside it. If the flux surface $\Omega$ is held fixed, the function

$$
X(\Omega, \theta)= \pm \sqrt{\frac{\Omega+\cos \theta}{8}}
$$

has the following averages over $\theta$,

$$
\bar{X}=\frac{1}{\pi}[E(m)-(1-m) K(m)], \quad m<1,
$$




$$
\bar{X}=\frac{\sqrt{m}}{\pi} E\left(m^{-1}\right), \quad m>1,
$$

inside and outside the island, respectively, where $E$ and $K$ are elliptic integrals. Because of incompressibility, the runaway electrons that originally (i.e., before the island formed) occupied the space $-X_{0}<X<X_{0}$ end up within the flux surface $\Omega$ satisfying $\bar{X}(\Omega)=$ $X_{0}$, see Fig. 1. Because of freezing-in, if the pre-island current profile was

$$
j_{0}=j_{0}(0)+\frac{1}{2} j_{0}^{\prime \prime}(0) X^{2},
$$

then the final current profile becomes

$$
j_{\dagger}=j_{0}(0)+\frac{1}{2} j_{0}^{\prime \prime}(0) \bar{X}^{2} .
$$

Before reconnection, the flux surfaces are straight, i.e., they are surfaces of constant $X$, while after reconnection they are surfaces of constant $\bar{X}(x, y)$. The point is that, because of incompressibility and freezing in, the runaway electrons intially on a flux surface labelled by $X$ are mapped to the post-reconnection surface for which $\bar{X}(x, y)=$ $X$. The resulting current profile (50) is different from that arising in the corresponding situation with Ohmic current, where the profile is instead determined by the fluxsurface-averaged Ohm's law $[9,12,13,16]$. The island saturation amplitude is therefore also different.

In order to calculate this amplitude, we follow Ref. [9] and match the inner solution (42) to the outer, ideal-MHD solution, by demanding that the function

$$
M(x)=\frac{1}{\pi \psi_{0}} \int_{-x}^{x} d x^{\prime} \int_{-\pi}^{\pi} \frac{\partial^{2} \psi}{\partial x^{2}} \cos \theta d \theta=-\frac{w}{\pi \psi_{0}} \int_{-X}^{X} d X^{\prime} \int_{-\pi}^{\pi} \cos \theta\left(j_{\dagger}+\frac{\partial^{2} \psi_{\dagger}}{\partial \theta^{2}}\right) d \theta
$$

should for $X>>1$ match the corresponding quantity calculated using the exterior solution. Using the current profile (50), we obtain

$$
M_{\text {inner }}=-\frac{w j_{0}^{\prime \prime}(0)}{\pi j_{0}(0)} \int_{-1}^{\Omega} d \Omega^{\prime} \int_{-\theta_{0}}^{\theta_{0}} \frac{\bar{X}^{2} \cos \theta}{X} d \theta
$$

where $\theta_{0}(\Omega)$ denotes the maximum value of the angle $\theta \leq \pi$ on the flux surface $\Omega$, so that $\cos \theta_{0}=-\Omega$ inside the island and $\theta_{0}=\pi$ outside the island. The contribution to the double integral from the region inside the island is

$$
\int_{-1}^{1} \bar{X}^{2} d \Omega \int_{-\theta_{0}}^{\theta_{0}} \frac{\cos \theta}{X} d \theta=\frac{16}{\pi^{2}} \int_{0}^{1}[2 E(m)-K(m)][E(m)-(1-m) K(m)]^{2} d m \simeq-0.0262,
$$


and that from the outside becomes

$$
\int_{0}^{\Omega} \bar{X}^{2} d \Omega^{\prime} \int_{-\theta_{0}}^{\theta_{0}} \frac{\cos \theta}{X} d \theta=\frac{32}{\pi^{2}} \int_{\mu}^{1} E^{2}\left(\mu^{\prime}\right)\left[E\left(\mu^{\prime}\right)-\left(1-\frac{\mu}{2}\right) K\left(\mu^{\prime}\right)\right] \frac{d \mu^{\prime}}{\mu^{\prime 7 / 2}},
$$

with $\mu=1 / m$. This integral diverges for $\mu \rightarrow 0$, i.e., when taken over an area much larger than the island. In this limit

$$
\int_{0}^{\Omega} \bar{X}^{2} d \Omega^{\prime} \int_{-\theta_{0}}^{\theta_{0}} \frac{\cos \theta}{X} d \theta \rightarrow-0.297+\frac{3 \pi}{8}-\pi X
$$

where the first term results from splitting off the dominant diverging dependence from the integrand:

$$
\frac{32}{\pi^{2}} \int_{\mu}^{1}\left\{E^{2}\left(\mu^{\prime}\right)\left[E\left(\mu^{\prime}\right)-\left(1-\frac{\mu}{2}\right) K\left(\mu^{\prime}\right)\right]+\frac{\pi^{3} \mu^{\prime 2}}{128}\left(1+\frac{\mu^{\prime}}{4}\right)\right\} \frac{d \mu^{\prime}}{\mu^{\prime 7 / 2}} \simeq-0.297 .
$$

We thus conclude that the inner limit of the function (52) is

$$
M_{\mathrm{inner}} \rightarrow \frac{w j_{0}^{\prime \prime}(0)}{j_{0}(0)}(X-0.272)
$$

which is to be matched to the outer limit, obtained from the ideal MHD equation

$$
\left[\psi_{0}, \nabla_{\perp}^{2} \psi_{1}\right]+\left[\psi_{1}, \nabla_{\perp}^{2} \psi_{0}\right]=0
$$

where $\psi_{1}=\psi_{\dagger}-\psi_{0}$. For $\psi_{1} \propto \cos k y$, this equation becomes

$$
\frac{d^{2} \psi_{1}}{d x^{2}}+\left(\frac{d j_{0} / d x}{d \psi_{0} / d x}-k^{2}\right) \psi_{1}=0
$$

and has for a symmertric equilibrium the solution [14]

$$
\psi_{1}(x, y) \propto\left(1+\frac{1}{2} \Delta^{\prime}|x|+\frac{k^{2}+b}{2} x^{2}+\ldots\right) \cos k y
$$

where

Hence

$$
b=\left.\frac{\psi_{0}^{(4)}}{\psi_{0}^{\prime \prime}}\right|_{x=0}
$$

$$
M_{\text {outer }} \rightarrow \Delta^{\prime}+2\left(k^{2}+b\right) x+\ldots
$$

and matching with the inner solution implies that the saturated island size must be

$$
w=-\frac{j_{0}(0)}{j_{0}^{\prime \prime}(0)} \frac{\Delta^{\prime}}{0.272}
$$

In contrast, the saturation amplitude in a purely resistive MHD plasma is [14]

$$
w=-\frac{j_{0}(0)}{j_{0}^{\prime \prime}(0)} \frac{\Delta^{\prime}}{0.411}
$$


which is smaller than (63). It comes as no surprise that these results are different since the saturation mechanisms are different. In both cases, the island grows until the magnetic energy of the system is no longer lowered by further growth, subject to the constraints that the current must obey. In the runaway case these constraints come from the requirement of freezing-in, while in the resistive case they originate from the flux-surface average of Ohm's law.

\section{Numerical results}

In order to confirm the analytical results of the two preceding sections, and to further explore the growth of magnetic islands in plasmas with and without runaway electrons, we have carried out direct numerical simulations of nonlinear equations (4), (10) and (12). These equations were solved as an initial value problem in the geometry of a sheared slab, with an equilibrium current profile proportional to $\cos x$ and no equilibrium electric field. The simulations were carried out with an initial value code, based on a Fast Fourier Transform scheme for the space operations and on a third order AdamsBashfort scheme for advancing the equations in time. All the simulations reported here were performed using a grid of $1024 \times 64$ points. The choice of this grid has been justified by convergence tests at higher resolution. The simulations were initialized with a perturbation on the current density profile given by

$$
j_{1}(x, y)=\hat{J}(x) \exp \left(i k_{y} y\right)
$$

where $\hat{J}(x)$ is a function localized around the rational surface, within a width of order of the linear layer and a typical amplitude of $10^{-4}$.

Numerically calculated linear growth rates are shown in Fig. 2, where results are included both for the case where the equilibrium current is Ohmic and where it is carried by runaway electrons. Also shown are the analytical predictions of FurthKilleen-Rosenbluth [5], and the finite-resistivity correction due to Militello et al [7]. It is clear that the former agrees with the runaway simulations and the latter with the Ohmic simulations, as predicted analytically in the Appendix.

The nonlinear island growth is shown as a function of time in Figs. 3 and 4 in the case of an Ohmic and a runaway plasma, respectively. The agreement with the analytical predictions of the saturation amplitude, $\Delta^{\prime}=0.411 w$ in the Ohmic case and $\Delta^{\prime}=$ 
$0.272 w$ in the runaway case, is good for small values of $\Delta^{\prime}$ and gets progressively worse as this parameter increases. This is not surprising since the calculation of the saturated state assumes that the island is small, i.e., $w \sim \Delta^{\prime} \ll 1$. Analytical considerations suggest that the next term in the $w$-expansion should be of order $w^{3}$, and this term can in principle be calculated along the lines laid out in Sec. IV and Refs. [9, 12, 13, 16]. However, this calculation is fairly lengthy as it involves determining the shape of the island to higher accuracy than the single-harmonic approximation that we used. Instead, we have determined the coefficient of the $w^{3}$ term by fitting to our numerical results, and obtained the saturation formula

$$
\Delta^{\prime}=0.27 w-0.013 w^{3}
$$

which agrees well with the numerical simulations shown in Fig. 5.

However, this formula also leads to an interesting prediction, namely, that no saturation should occur above a critical value of $\Delta^{\prime} \simeq 0.47$. Figure 6 shows the numerically determined saturation amplitude versus $\Delta^{\prime}$ and also suggests a nonlinear bifurcation at this value of $\Delta^{\prime}$. When we perform simulations at larger values, we find that the island grows quietly for a while, and then suddently enters a period of explosive growth until it fills up the entire computational domain. An example of this behavior is given in Fig. 7. When considering the final part of the island evolution in these calculations, one must remember that the island is getting so wide that it becomes influenced by boundary conditions. In the present example with an equilibrium flux proportional to $\cos x$, periodic boundary conditions apply. There are consequently neighbouring islands at $x= \pm \pi$, which interact with the one located at $x=0$ when the island size is of the order of the equilibrium shear length. The evolution of the island beyond the bifurcation point is therefore uncertain and dependent on global aspects of the computational domain. It should be mentioned that a nonlinear bifurcation of the saturation amplitude has earlier been found for Ohmic plasmas [17], where however the critical value of $\Delta^{\prime}$ was much larger. We also point out that the bifurcation is sensitive to the equilibrium geometry, and we have only considered the slab here. In a cylinder, one expects a term of order $w^{2}$ to be present in Eq. (65), which will of course change the behaviour qualitatively.

It is also intersting to compare the runaway current denisity in the simulations with the analytical prediction (50). Such a comparison is shown in Fig. 8, where the current 
density perturbation in the saturated state is shown as a function of $x$ along a line of constant $y$ passing through the O-point of the island. The agreement is excellent inside the separatrix (located at $x=0.37$ ) but outside the island there is some discrepancy (less than about 10\%) for reasons we have not been able to identify. More importantly, the result is very different from that obtained when the current is taken to be Ohmic (not shown), in which case the perurbation is only about half is big.

\section{Conclusions}

We have established that in a plasma where the current is carried by collisionless runaway electrons but the bulk plasma is governed by resistive MHD, the linear properties of the tearing mode are approximately the same as if there were no runaways and the current were instead carried by thermal electrons. The linear growth rates are the same in leading order, but corrections due to finite resistivity are different. The linear analysis is valid when the magnetic island width is much smaller than the resistive layer width (which is of order $1 \mathrm{~cm}$ in a typical post-disruption plasma). We have assumed that all the equilibrium plasma current is carried by the runaway electrons, which is true several resistive diffusion times after the thermal quench of a disruption. In practice, islands may form before all the toroidal electric field has diffused out of the plasma, so that some of the plasma current is still carried by thermal electrons. Since the growth rate (2) is much larger than the inverse skin time of the plasma column, such islands would quickly enter the nonlinear regime and then experience nonlinear growth until the thermal plasma current has subsided.

Although the linear properties of magnetic islands are approximately the same in plasmas with and without runaway electrons, the nonlinear saturation amplitude is different. In the simplest case of a symmetric slab equilibrium, the saturated island width is about $50 \%$ larger in a runaway plasma. Above a critical value of the stability index, the saturation amplitude undergoes a nonlinear bifurcation and suddenly jumps to a larger value. 


\section{Acknowledgment}

We are grateful to Chris Gimblett for many helpful discussions during the initial course of this work. This work was partly supported by the Euratom Communities under contracts of Association between EURATOM and ENEA, UKAEA and IPP, respectively. The views and opinions expressed herein do not necessarily reflect those of the European Commission. 


\section{Appendix: Linear growth rate}

In this Appendix, we calculate the linear growth rate for instability, in the case of slab geometry, in a plasma where the current is either carried by thermal or runaway electrons. The Fourier transform,

$$
\hat{\varphi}^{(0)}(q)=\int_{-\infty}^{\infty} \varphi^{(0)}(x) e^{-i q x} d x,
$$

of Eq. (34) is

$$
\frac{d^{2} \hat{\varphi}^{(0)}}{d q^{2}}-q^{2} \hat{\varphi}^{(0)}=A \delta^{\prime}(q)
$$

and has the solution

$$
\hat{\varphi}^{(0)} \propto U(0, q \sqrt{2})
$$

for $q>0$, where $U$ denotes a parabolic cylinder function. For small $q$ this solution reduces to

$$
\hat{\varphi}^{(0)}(q) \propto 1-\frac{\sqrt{2} \Gamma(3 / 4)}{\Gamma(1 / 4)} q .
$$

and is to be matched to the exterior, ideal MHD region, where the perturbed magnetic flux is given by Eq. (59). In order to retain the most important corrections due to finite resistivity, we follow Ref. [7] and replace Eq. (20) by the more accurate relation

$$
i k_{\|}^{\prime} x \phi=\eta\left(\nabla_{\perp}^{2} \psi+\lambda j\right)-\gamma \psi \simeq A\left[\eta \Delta^{\prime} \delta(x)+\eta b-\gamma\right]+\lambda \eta j,
$$

where we have used Eq. (59) in the second, approximate inequality, and where $\lambda=0$ if the equilibrium current is Ohmic and $\lambda=1$ if it is carried by runaway electrons. To lowest order in $v_{A} / c \ll 1$, the runaway equation (12) becomes in a symmetric slab

$$
\left[\psi_{0}+\psi_{1}, j_{0}+j_{1}\right]=i k e^{i k y}\left(\psi_{0}^{\prime} j-j_{0}^{\prime} \psi\right)=0
$$

where we have written $\psi_{1}=\psi(x) e^{i k y}$ and $j_{1}=j(x) e^{i k y}$, i.e.,

$$
j=-\psi b,
$$

for resonance at $x=0$. Hence

$$
i k_{\|}^{\prime} x \phi \propto \eta \Delta^{\prime} \delta(x)+(1-\lambda) \eta b-\gamma
$$

which we proceed to Fourier transform, obtaining

$$
\frac{d \hat{\varphi}^{(0)}}{d q} \propto w[\gamma-(1-\lambda) \eta b] \delta(q)-\frac{\Delta^{\prime} \eta}{2 \pi},
$$


where we recall that $x=w z$, with $w=(\eta \gamma)^{1 / 4} / k_{\|}^{\prime 1 / 2}$. Integrating this relation across $q=0$ and using the fact that $\hat{\varphi}^{(0)}$ is odd gives

$$
2 \hat{\varphi}^{(0)}(q)=w[\gamma-(1-\lambda) \eta b]-\frac{\Delta^{\prime} \eta q}{\pi} .
$$

Finally, the growth rate is obtained by comparing this result with the inner solution (69),

$$
w[\gamma-(1-\lambda) \eta b]=\frac{\Gamma(1 / 4) \eta \Delta^{\prime}}{2 \pi \Gamma(3 / 4)} .
$$

If $\lambda=1$, this gives the standard Furth-Killeen-Rosenbluth (FKR) growth rate [5], and if $\lambda=0$ it yields the correction calculated by Militello et al [7]. The latter is thus absent in a plasma where the current is carried by runaway electrons. 


\section{References}

[1] L.-G. Eriksson, P. Helander, F. Andersson, D. Anderson, and M. Lisak, Phys. Rev. Lett. 92, 205004 (2004).

[2] M.N. Rosenbluth and S.V. Putvinski, Nucl. Fusion 37, 1355 (1997).

[3] R.D. Gill, B. Alper, A.W. Edwards, L.C. Ingesson, M.F. Johnson, and D.J. Ward, Nucl. Fusion 40, 163 (2000).

[4] H.R. Strauss, Phys. Fluids 19, 134 (1976).

[5] H.P. Furth, J. Killeen, and M.N. Rosenbluth, Phys. Fluids 6, 459 (1963).

[6] G. Bertin, Phys. Rev. A 25, 1786 (1982).

[7] F. Militello, G. Huysmans, M. Ottaviani, and F. Porcelli, Phys. Plasmas 11, 125 (2004).

[8] P.H. Rutherford, Phys. Fluids 16, 1903 (1973).

[9] F. Militello, R.J. Hastie, and F. Porcelli, Phys. Plasmas 13, 112512 (2006).

[10] R.D. Hazeltine and J.D. Meiss, Plasma Confinement (Addison-Wesley, Redwood City, 1992), p. 290.

[11] R.B.White,D.A.Monticello, M.N.Rosenbluth and B.V.Waddell, Phys. Fluids, 20, 800 (1977).

[12] A.Thyagaraja, Phys. Fluids, 24, 1716 (1981).

[13] N. Arcis, D. Escande and M. Ottaviani, Phys. Letts. A 347, 241 (2005); Phys. Plasmas 13, 052305 (2006).

[14] F. Militello and F. Porcelli, Phys. Plasmas, 11, L13 (2004).

[15] D.F. Escande and M. Ottaviani, Phys. Lett. A 323, 278 (2004).

[16] R.J. Hastie, F. Militello and F. Porcelli, Phys. Rev. Lett., 95, 065001 (2005).

[17] C. Tebaldi, M. Ottaviani, and F. Porcelli, Plasma Phys. Contr. Fusion 38, 619 (1996). 


\section{Figure captions}

Fig. 1 (Color online) Two pre-reconnection field lines (dashed) and three post-reconnection field lines (solid) are shown, the latter corresponding to $\Omega=1 / 4, \Omega=1$, and $\Omega=5 / 2$, respectively. The runaway electrons are frozen into the field, and those who were located between the two dashed lines (dashed region, yellow online) before reconnetion end up in the area $\Omega<1 / 4$ after reconnection has occurred. These regions have equal area.

Fig. 2 Numerically calculated linear growth rates for magnetic islands in a plasma with $(*)$ and without $(\diamond)$ runaway electrons; analytical result of Furth-KilleenRosenbluth (solid line) and Militello et al. (dashed line).

Fig. 3 Nonlinear growth of magnetic islands in an Ohmic plasma, for $\Delta^{\prime}=0.2$ and $\Delta^{\prime}=0.3$. The island widths have been normalized to the theoretical prediction $w=2.44 \Delta^{\prime}$.

Fig. 4 Nonlinear growth of magnetic islands in a plasma where the current is carried by runaway electrons, for a range of different values of $\Delta^{\prime}$. The island widths have been normalized to the theoretical prediction $w=\Delta^{\prime} / 0.27$

Fig. 5 Nonlinear island growth in a runaway plasma. The quantity $\left(0.27 w-0.013 w^{3}\right) / \Delta^{\prime}$ is plotted as a function of time.

Fig. 6 Saturated island width vs $\Delta^{\prime}$. Note that the slope becomes infinite at $\Delta^{\prime} \simeq 0.44$

Fig. 7 Island width vs time in a runaway plasma with $\Delta^{\prime}=0.48$.

Fig. 8 Runaway current density perturbation in the saturated state along a line of constant $y$ through the island O-point, $\Delta^{\prime}=0.2$. 


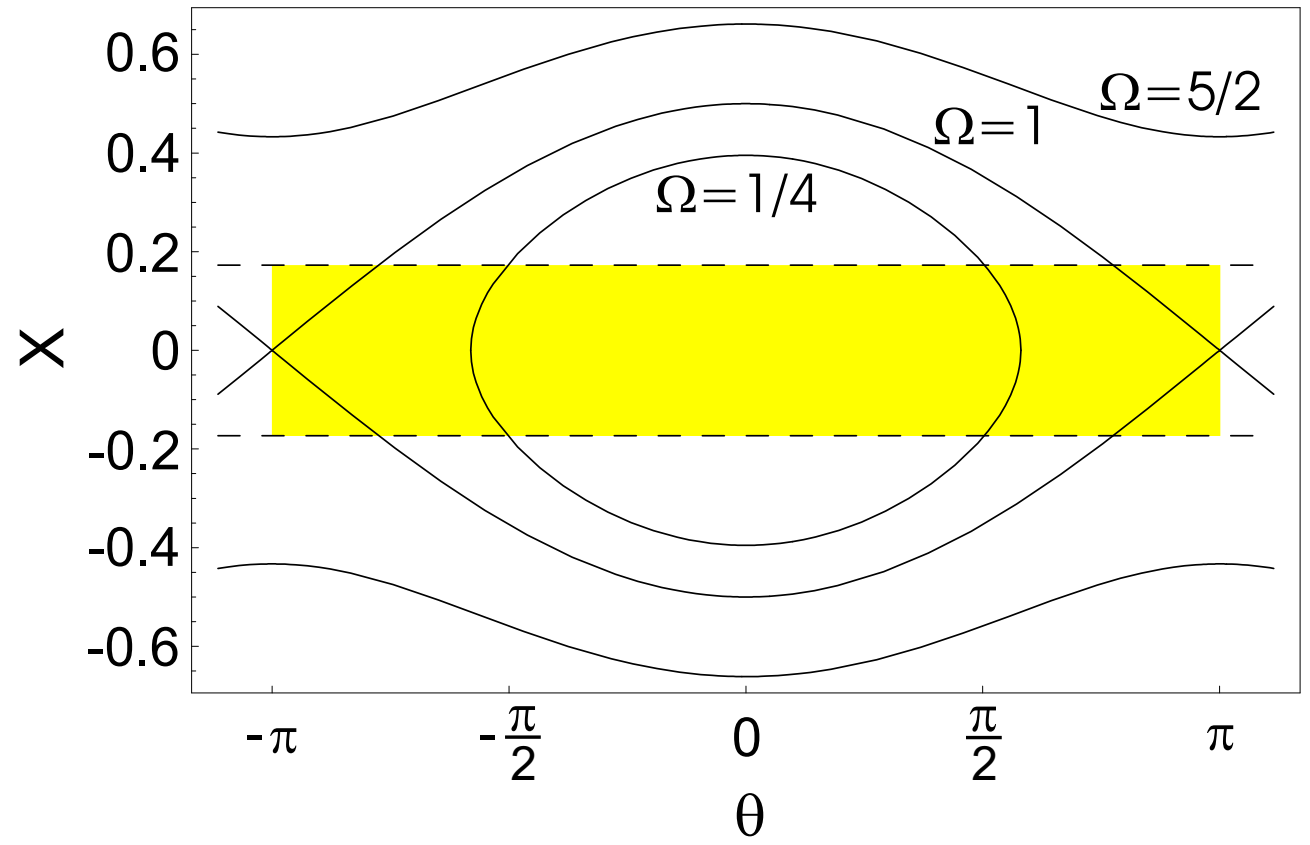

Figure 1: 


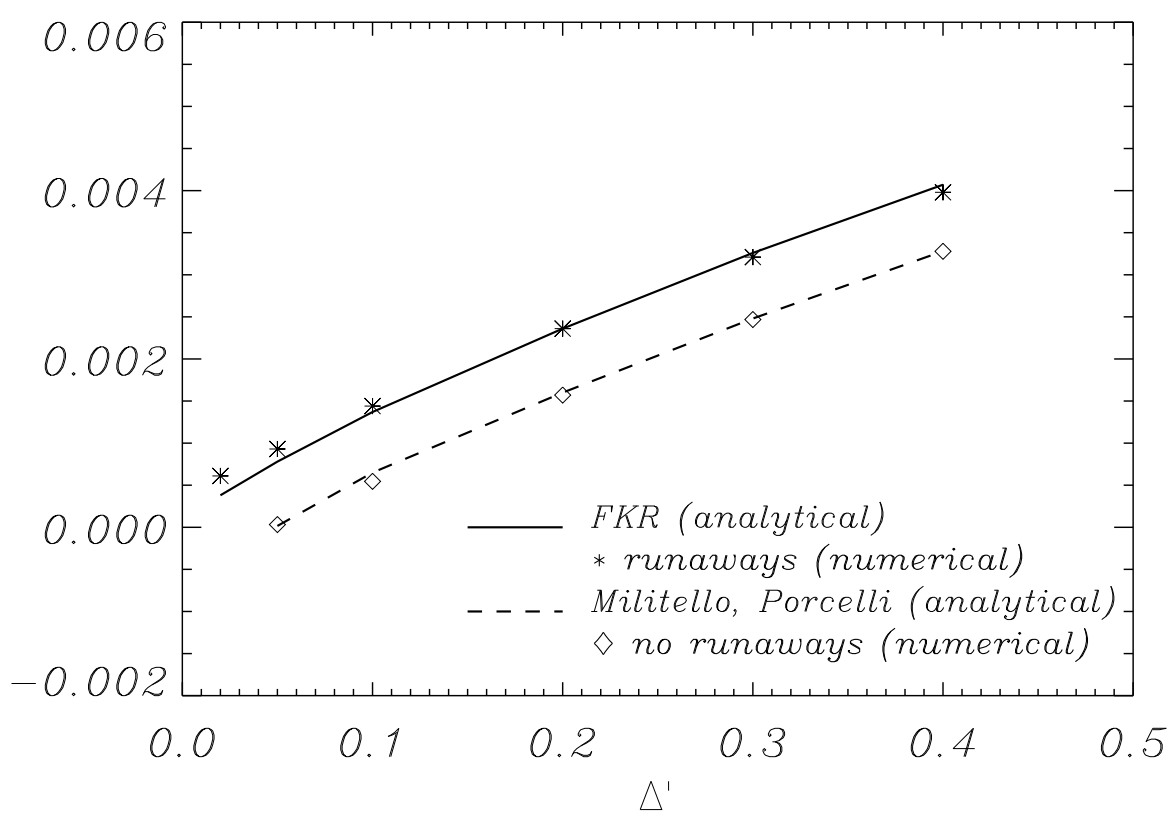

Figure 2: 


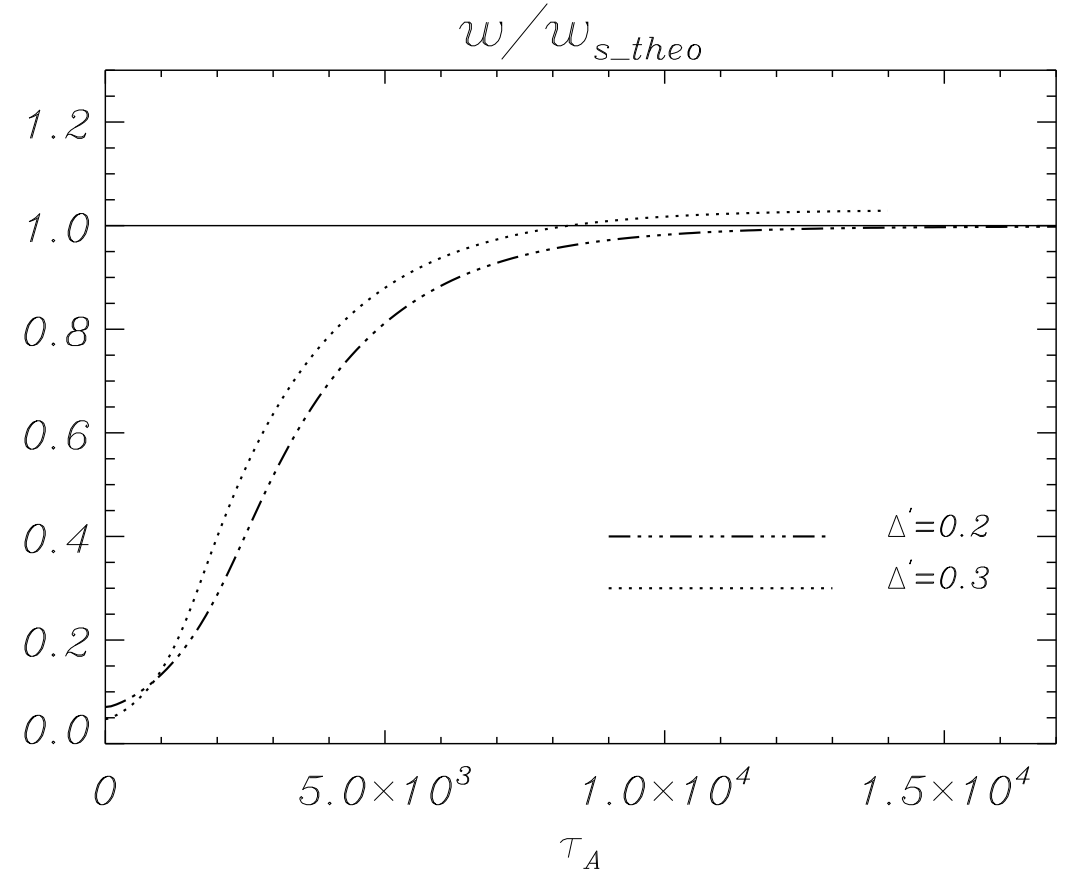

Figure 3: 


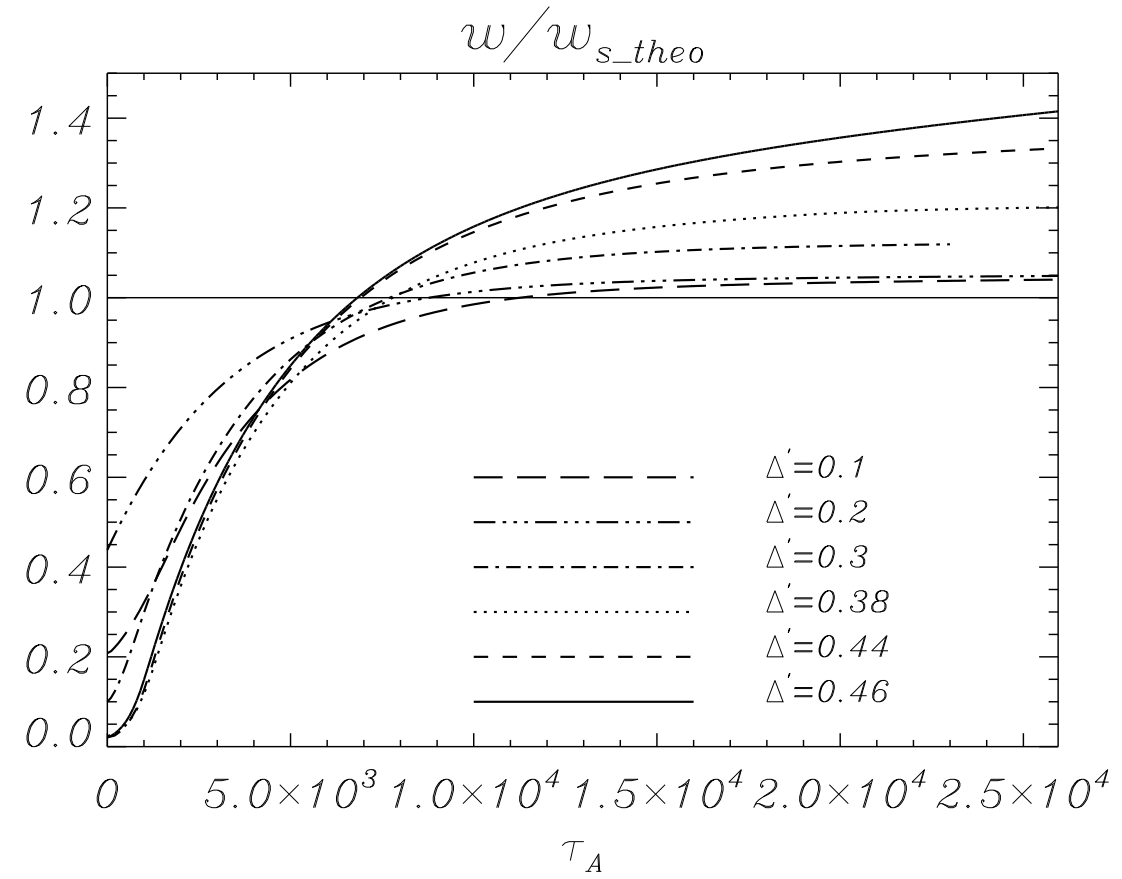

Figure 4: 


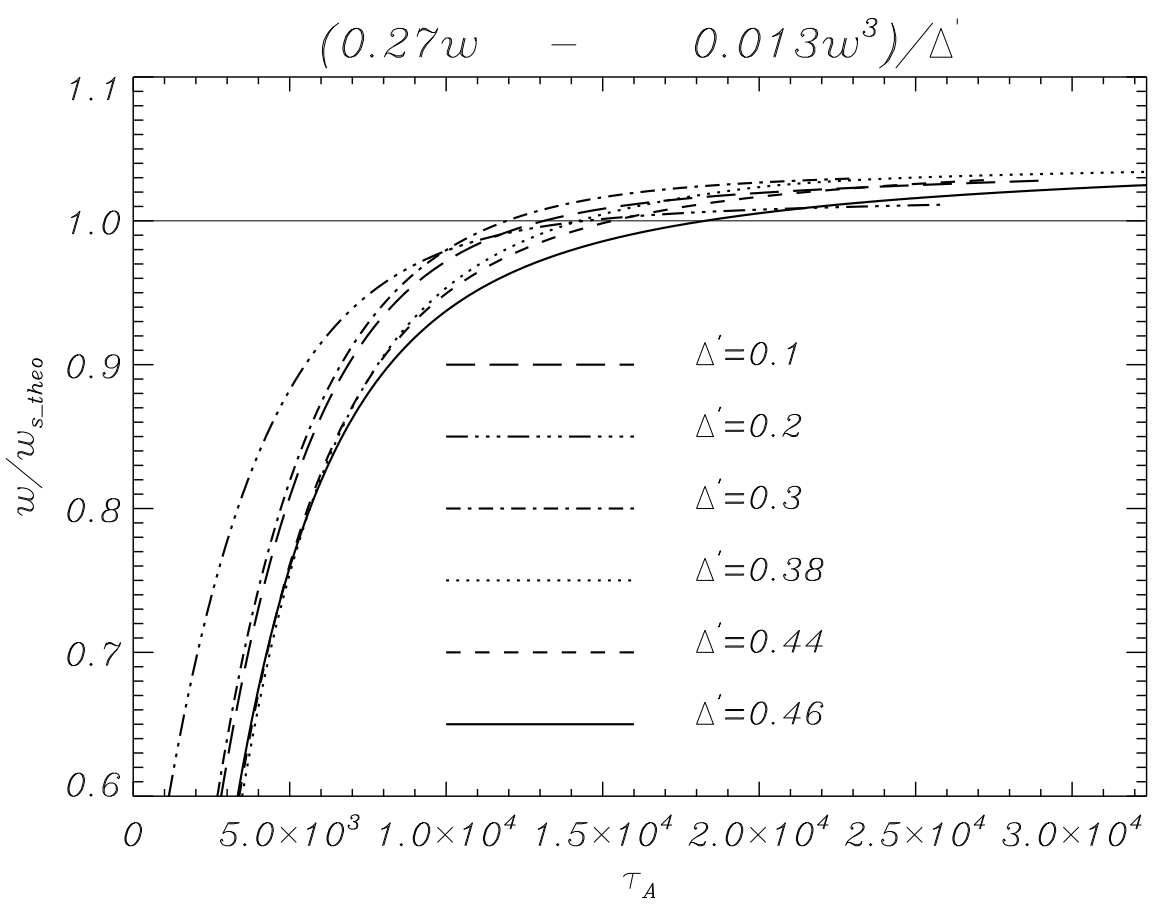

Figure 5: 


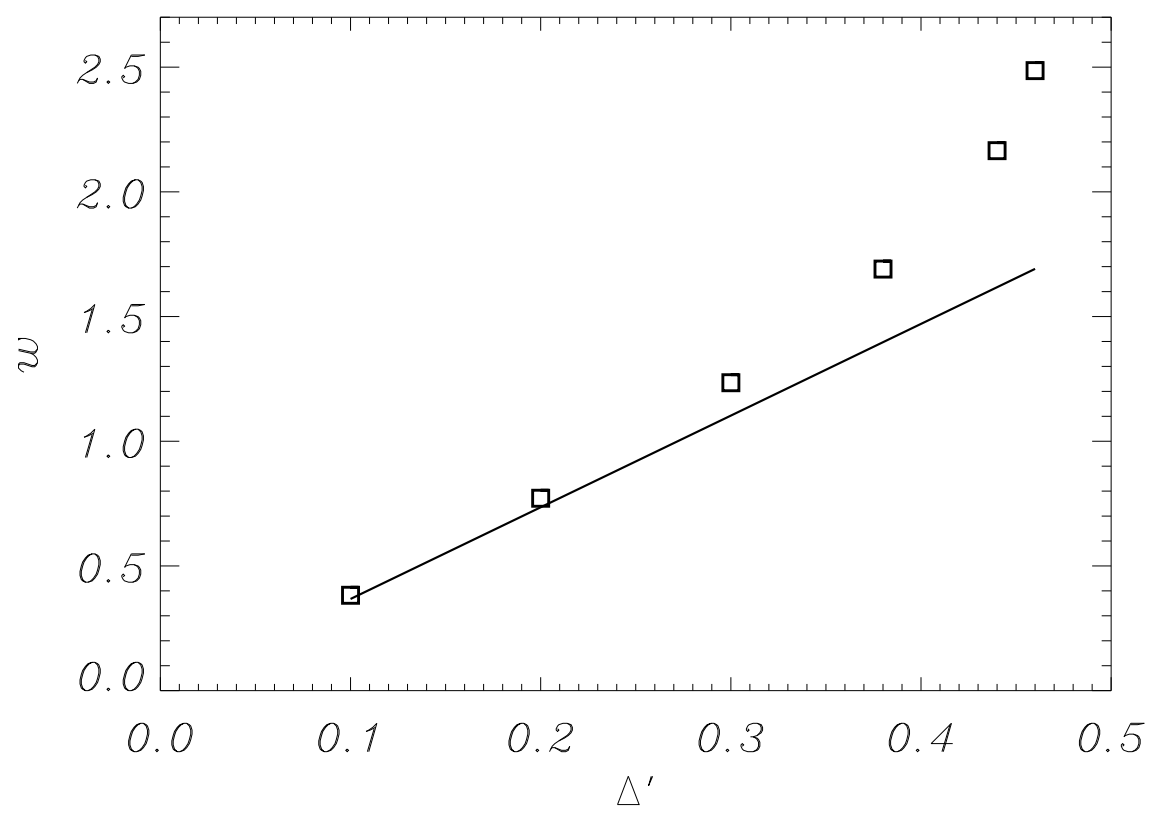

Figure 6: 


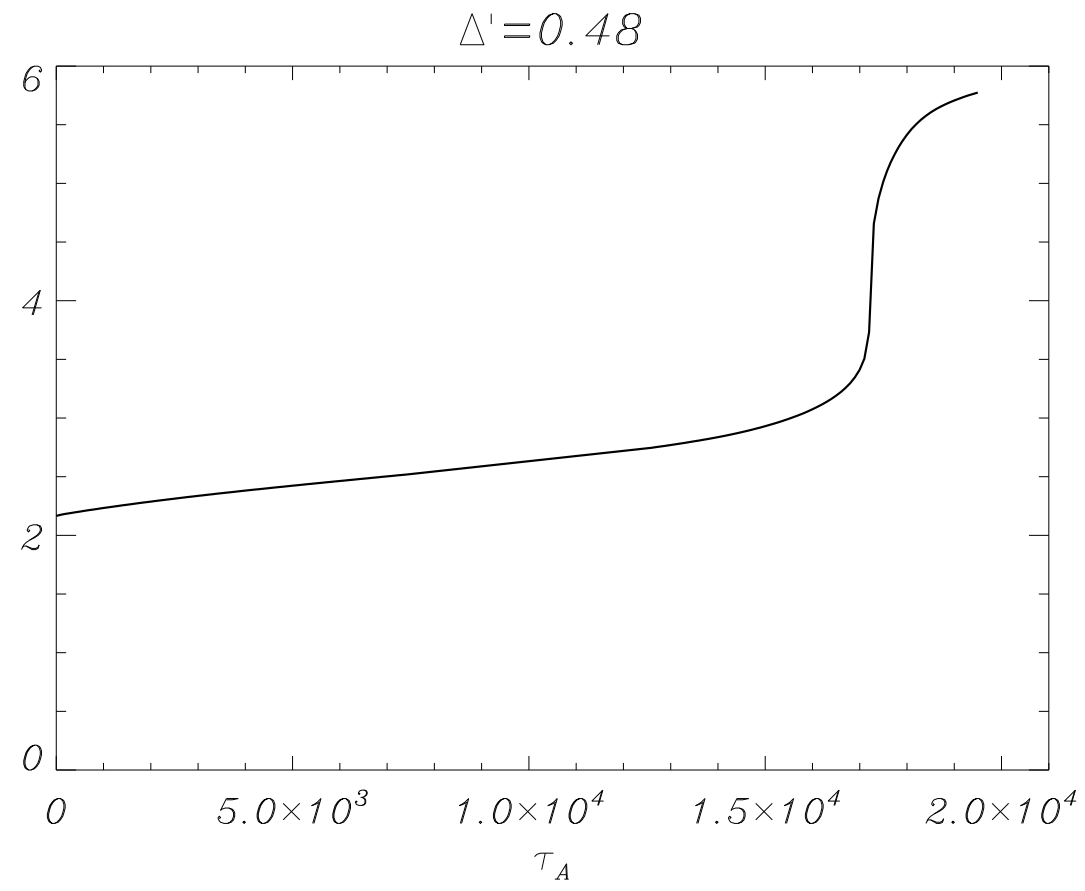

Figure 7: 


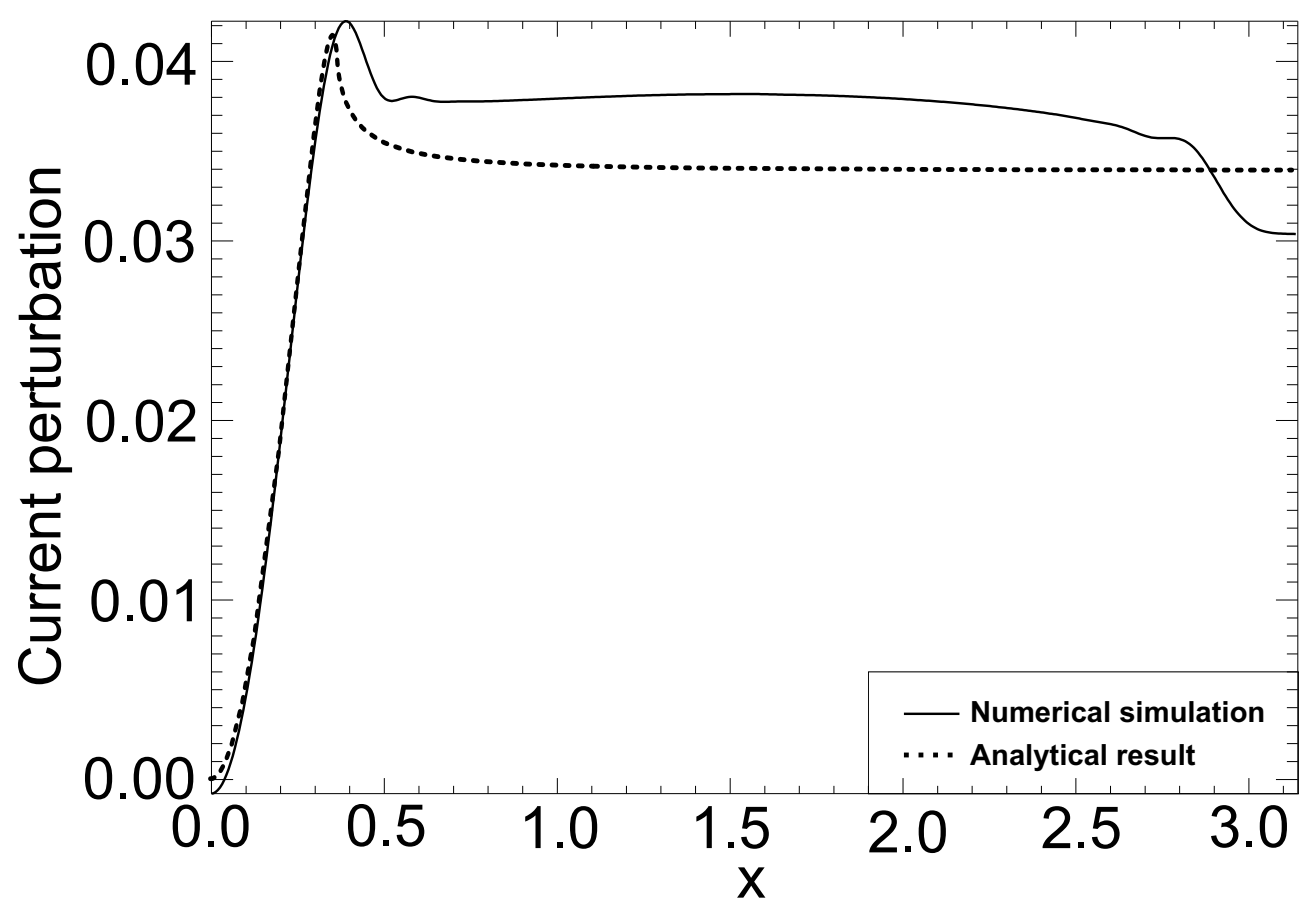

Figure 8: 